\title{
A Fully Integrated Magnetically Actuated Micromachined Relay
}

\author{
William P. Taylor and Mark G. Allen \\ Georgia Institute of Technology, School of Electrical and Computer Engineering \\ Atlanta, GA 30332-0250 \\ Charles R. Dauwalter \\ Milli Sensor Systems and Actuators \\ 93 Border Street, W. Newton, MA 02165-2013
}

\begin{abstract}
A fully integrated magnetically actuated micromachined relay (microrelay) has been fabricated and tested. Fabricated devices range in size from $6 \mathrm{~mm} \times 7 \mathrm{~mm}$ to $7 \mathrm{~mm} \times 7.4 \mathrm{~mm}$. A coil current of $600 \mathrm{~mA}$ actuates the microrelay, with a coil power dissipation of $320 \mathrm{~mW}$. The $\mathrm{ON}$ resistance of the microrelays is typically less than $30 \Omega$, with values as low as $0.85 \Omega$ observed under electromagnetic operation. A contact resistance of $0.017 \Omega$ was observed under mechanical actuation.
\end{abstract}

\section{INTRODUCTION}

Due to parasitic effects and offset voltage injection in solid state relays, electromechanical relays remain widely used in a variety of applications such as data acquisition boards and aircraft and automotive control circuitry. Micromachined versions of these relays (microrelays) are desirable due to their potential for smaller size and batch fabrication.

Micromachined silicon switches were demonstrated in 1979 by Petersen [1]. These devices were bulk micromachined, electrostatically actuated, and contained metal contacts in order to reduce contact resistance and increase current carrying capacity. More recently, electrostatic polysilicon microrelays have been achieved $[2,3]$. These devices have a contact resistance of approximately $10 \mathrm{k} \Omega$, and a carry current of less than $10 \mathrm{~mA}$. More recent versions of electrostatically-driven microrelays with metallic contacts and lower contact resistance have also been reported [4-7]. These devices have the advantage of higher carry currents, and cycle lifetimes on the order of $10^{8}[5]$. Another recently-reported microrelay device employs the use of a mercury droplet to eliminate some of the contact resistance issues, such as contact force, wear, and oxidation [8]. This device was reported to have a contact resistance of less than $1 \Omega$, with a carry current of $20 \mathrm{~mA}$.

Lower actuation voltages may be useful in many applications, such as automotive applications in which lowvoltage, high current electrical systems are present. Magnetically-driven microrelays, which have lower actuation voltages, may satisfy these constraints. Previously reported magnetic microrelays [9-11] do not have fully integrated magnetic coils. Instead, these devices either require an external coil for electromagnetic actuation or integrated heating coils which act to demagnetize portions of an externally-driven magnetic circuit and results in a switching action. The use of a fully integrated magnetically actuated microrelay design combines the manufacturing, cost, and size benefits of full integration with the benefits of magnetic actuation. In addition, these devices offer the potential for plug-in replacement of conventional reed or other relays in current designs. Our approach is to use micromachining techniques to realize a magnetically-driven microrelay.

\section{DEVICE CONCEPT}

Figure 1 shows a schematic top view of the microrelay. The basic structure of the microrelay consists of a zigzag or meander coil which winds over an insulated lower magnetic core, made of a soft magnetic material, and through soft magnetic material side cores. The coil is electrically insulated from the magnetic materials. The entire coil and core structure, which is also electrically insulated on the top, can be thought of as an integrated driving electromagnet. There are two contacts on top of the electromagnet. In order to achieve a relay action, the two contacts are alternately electrically connected and disconnected by a movable conducting platform, which is integrated with the structure using surface micromachining.

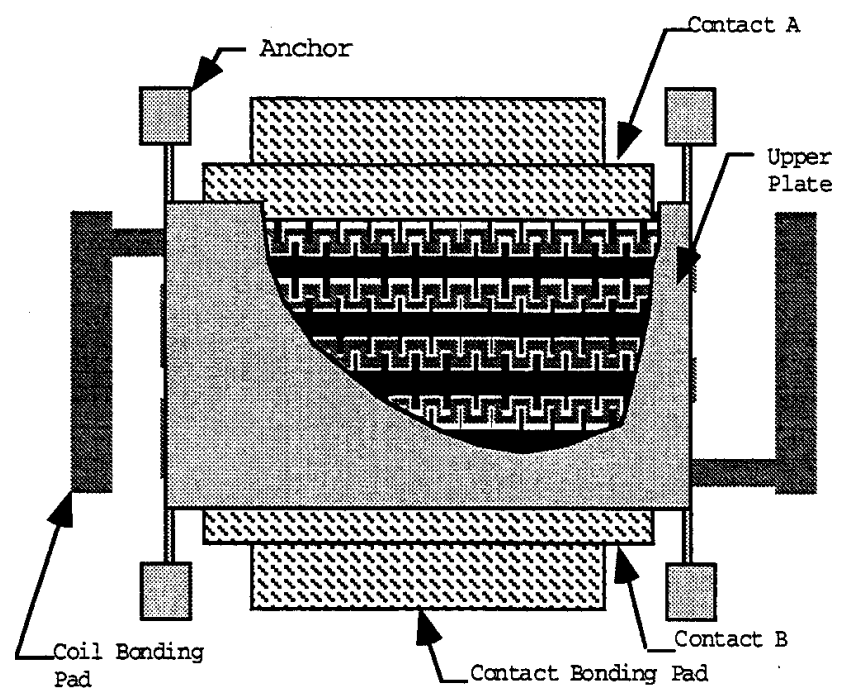

Figure 1: Schematic drawing of microrelay (top view)

The basic operation of the device is as follows. A current flows through the coil and generates a magnetic flux which creates a force on the upper magnetic movable plate. This force causes the upper plate to move downwards and connect the two contacts which results in a switching action. When the coil current is turned off, the mechanical stress in the arms generated by the downward movement of the plate forces the upper magnetic plate to rise from the contacts, thus opening the relay. Figure 1 shows a four-beam upper plate support configuration; however, other devices with varying beam numbers and geometries (including cantilever-type devices) have also been realized. 


\section{THEORY}

A cross section of the generalized actuator is shown in Figure 2. Each of the slots of the structure contains $\mathrm{N}$ electrical conductors, each of which carries current I. Assuming linearity, an expression for the normal (y-direction) force between the stator and armature may be obtained as follows. For the actuator being described, the width of a tooth, a, and the actuator length, 1 , are both relatively large compared to the gap, g, so that the effects of fringing are small and are neglected in the analysis. The permeability of the stator and armature iron is assumed to be large enough that the reluctance of the iron can be neglected.
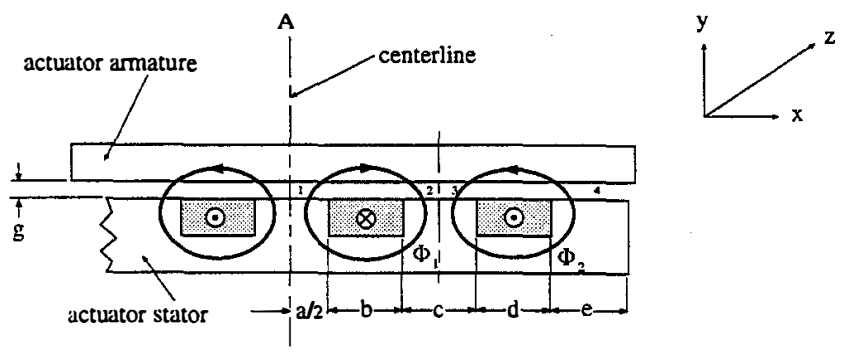

Figure 2: Side view of theoretical actuator with definitions of dimension variables included.

The magnetic reluctances of the air gap regions numbered 1,2 , 3,4 in the figure are:

$$
\begin{aligned}
& \Re_{2}=\Re_{3}=\frac{g}{\mu_{0}(c / 2) l}=\frac{2 g}{\mu_{0} c l} \\
& \Re_{1}=\frac{g}{\mu_{0}(a / 2) l}=\frac{2 g}{\mu_{0} a l} \text { and } \Re_{4}=\frac{g}{\mu_{0} e l}
\end{aligned}
$$

The combined permeance of air gap regions 2 and 3 is:

$$
P_{2,3}=\frac{1}{\Re_{2}}+\frac{1}{\Re_{3}}=\frac{\mu_{0} c l}{2 g}+\frac{\mu_{0} c l}{2 g}=\frac{\mu_{0} c l}{g}
$$

Similarly:

$$
P_{1,4}=\frac{1}{\Re_{1}}+\frac{1}{\Re_{4}}=\frac{\mu_{0} a l}{2 g}+\frac{\mu_{0} e l}{g}=\frac{\mu_{0} l}{2 g} \cdot(a+2 e)
$$

The reluctances of regions 2,3 and 1,4 are then:

$$
\Re_{2.3}=\frac{1}{P_{2,3}}=\frac{g}{\mu_{0} c l} \text { and } \Re_{1,4}=\frac{1}{P_{1,4}}=\frac{2 g}{\mu_{0} l(a+2 e)}
$$

The total air gap reluctance is:

$$
\Re_{\text {total }}=\Re_{1,4}+\Re_{2,3}=\frac{2 g}{\mu_{0} l}\left\{\frac{1}{a+2 e}+\frac{1}{2 c}\right\}=\frac{2 g\{2 c+a+2 e\}(6)}{\left.\mu_{0} l \mid 2 c(a+2 e)\right\}}
$$

so that the total air gap permeance becomes:

$$
P_{\text {total }}=\frac{1}{\Re_{\text {total }}}=\frac{\mu_{0} l}{2 g}\left\{\frac{2 c(a+2 e)}{2 c+(a+2 e)}\right\}=\frac{\mu_{0} c l}{g} \frac{(a+2 e)}{(a+2 e)+2 c}
$$

The total magnetic flux is the magnetomotive force, $F=N I$, multiplied by the total air gap permeance, viz:

$$
\Phi_{\text {total }}=N I P_{\text {total }}
$$

The total flux is then:

$$
\Phi_{\text {total }}=N I P_{\text {total }} \equiv \frac{\mu_{0} N I c l(a+2 e)}{g(a+2 e)+2 c}
$$

The flux, $\lambda$, linking the conductors is:

$$
\lambda=N \Phi_{\text {total }}=\frac{\mu_{0} N^{2} I c l(a+2 e)}{g \quad(a+2 e)+2 c}
$$

The inductance is defined as the flux linkages per unit current:

$$
L=\frac{\lambda}{I}=\frac{\mu_{0} N^{2} c l(a+2 e)}{g(a+2 e)+2 c}
$$

The magnetic energy, $\mathrm{W}$, is:

$$
W=\frac{1}{2} L I^{2}=\frac{\mu_{0}(N I)^{2} c l \quad(a+2 e)}{2 g \quad(a+2 e)+2 c}
$$

The normal (in the y direction) force on this section of the armature is therefore:

$$
F_{y}=\frac{d W}{d y}=\frac{-\mu_{0}(N I)^{2} c l(a+2 e)}{2 g^{2}(a+2 e)+2 c}
$$

The equations derived above were used to estimate the force generated on the upper plate. The relay air gap was sized to be small compared with other characteristic lengths, in order to minimize the effects of leakage flux. Typical relay geometries considered in this paper had initial forces on the upper plate in the $1-10 \mathrm{mN}$ range as calculated using the above equations. This force must be used both to overcome support beam flexure resistance as well as provide contact closure. From [9], it was estimated that a contact closure force of at least $200 \mu \mathrm{N}$ is required for reliable relay operation; the compliance of the upper plate support beams was calculated using this criterion. The coils were designed to carry a minimum of twice the estimated actuation current. Contacts were designed to obtain low contact resistance through the use of large contact areas. For this relay prototype, no effort was made to optimize the contacts for maximum lifetime or minimum resistance.

\section{FABRICATION}

Fabrication is based on standard polyimide mold electroplating techniques [12]. Figure 3 depicts the major fabrication steps. The fabrication begins by deposition of a seed layer onto the substrate followed by electroplating of a 
permalloy lower magnetic core through a pattemed polyimide mold. The lower core is then insulated with polyimide, and a copper coil is electrodeposited and insulated. The polyimide between the coil lines is removed with a standard plasma etching process, and the magnetic side cores are deposited in these cavities using the bottom magnetic core as the electroplating seed layer, forming the electromagnet. The entire electromagnet is then further insulated and planarized. Figure 4 shows a close-up of the completed electromagnet prior to contact and movable plate deposition. Contacts are deposited above the electromagnet, and a photoresist sacrificial layer is patterned over the contacts and electromagnet. An electroplating seed layer is deposited over the photoresist, and the upper magnetic plate is electrodeposited through a second photoresist mold. All photoresist and seed layers are then removed to release the upper magnetic plate. A photomicrograph of the completed and released microrelay is shown in Figure 5.
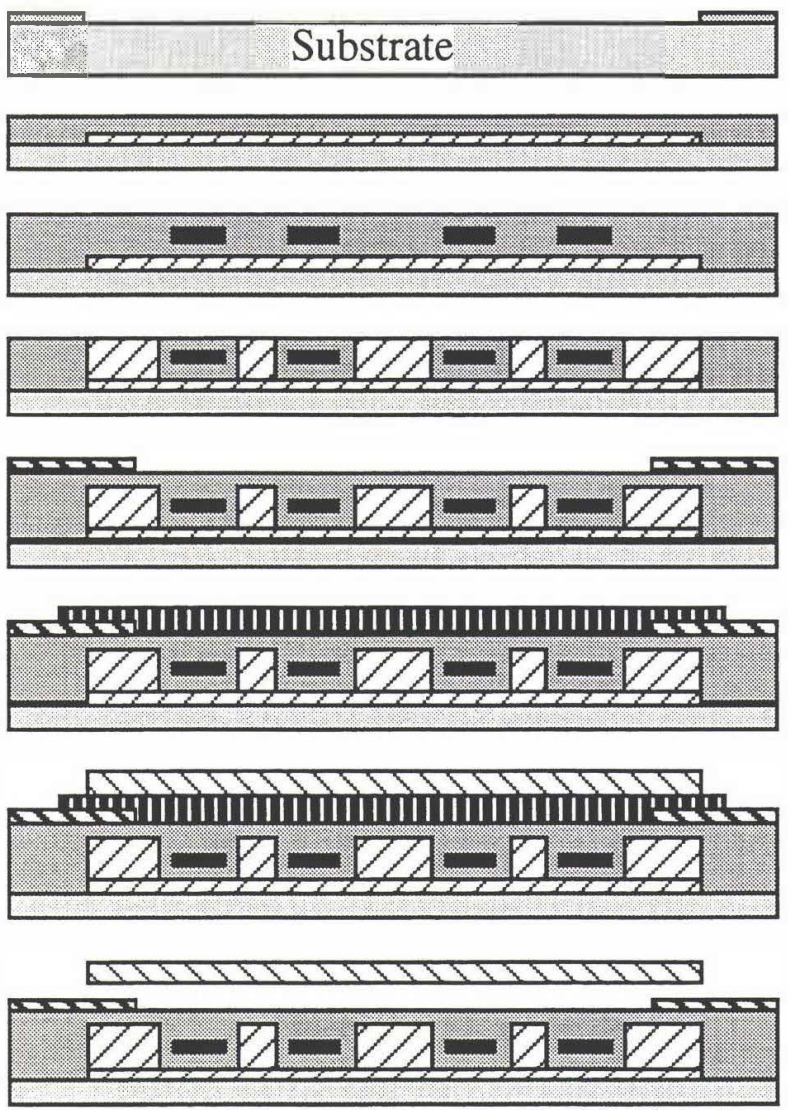

Nickel-Iron magnetic core

Movable Magnetic Upper Plate

Fixed Contacts
Coil Conductor

Polyimide

Sacrificial Layer
Figure 3: Fabrication sequence for the microrelay. (a) after deposition of plating mold for lower magnetic core; (b) after deposition of lower magnetic core; (c) after deposition of coil; (d) after formation of side cores; (e) after contact deposition; ( $f$ ) after sacrificial layer deposition; $(g)$ after deposition of upper magnetic plate; $(h)$ completed device.

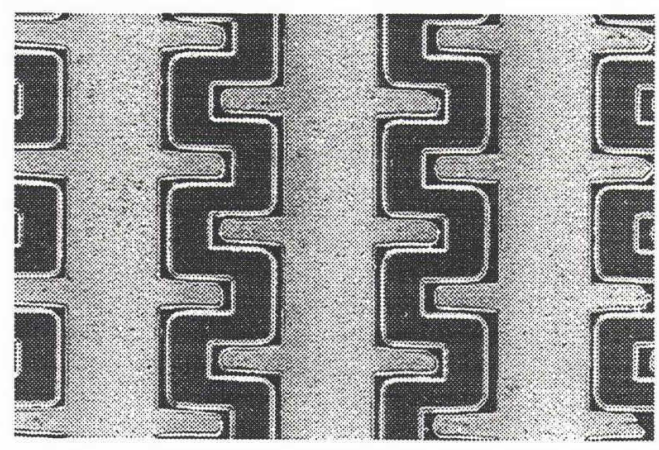

Figure 4: Photomicrograph of a fabricated electromagnet, top view. The darker serpentine regions are the coils; the light regions are the permalloy side cores.

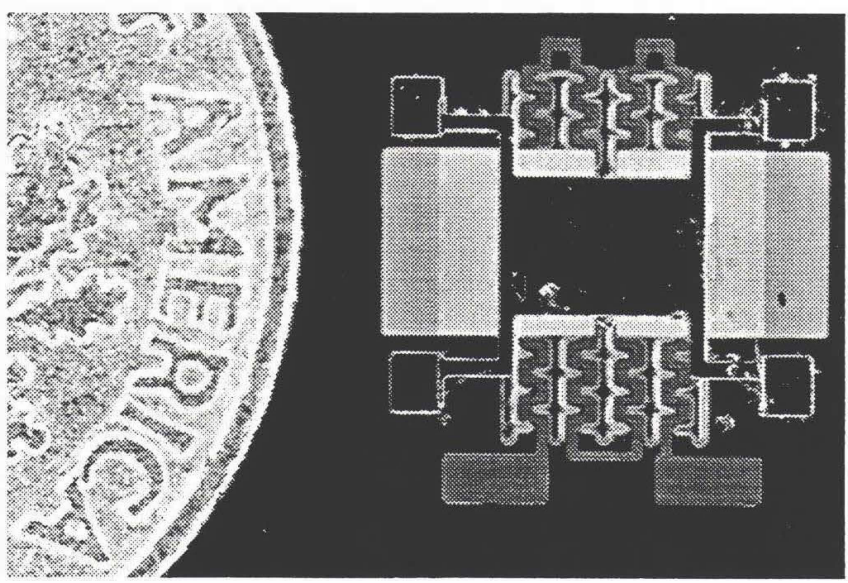

Figure 5: Photomicrograph of a completed magnetic microrelay, top view. The relay is shown next to a dime for comparison. The upper magnetic plate is suspended by four arms over the contacts. The upper magnetic plate is $2.2 \mathrm{~mm}$ by $3.4 \mathrm{~mm}$. The beams are $200 \mu \mathrm{m}$ in width, and $2 \mathrm{~mm}$ in length $(1 \mathrm{~mm}$ from plate to the turn in the beam, and $1 \mathrm{~mm}$ from the turn in the beam to the anchor point).

\section{RESULTS}

The average coil resistance was less than $1 \Omega$, depending on coil size and thickness. The microrelay coils have been tested up to 1.8A DC for several minutes and showed no signs of failure. Two types of plate suspension were tested: a four-arm bridge suspension as shown in Figure 5, and a two-arm cantilever type suspension (i.e., the relay as shown in Figure 5 but with the upper plate supported by two arms, both of which were on the same side of the suspended plate). The beam geometries for each type of suspension were varied. In addition, two operating modes of the relay were investigated: one in which the movable plate bridged both lower contacts, and one in which the movable plate formed one of the contacts and the lower contacts formed the second contact. The first operation mode has higher contact resistance but no current must flow through the movable plate support arms; the second operation mode has lower contact resistance since only one interface is formed upon relay closure. All types of suspensions and operating modes produced successfully actuating and functional relays. As expected, the more flexible cantilever-type suspension required lower actuation currents, and the second operation mode had lower contact resistance. For a typical cantilever-type device, with beam dimensions of $200 \mu \mathrm{m}$ width and $2 \mathrm{~mm}$ length, actuation was achieved with a $500 \mathrm{~mA}$ coil 
current, although the lowest contact resistances were observed at $600 \mathrm{~mA}$. For a bridge-type device, i.e., with four suspension arms, actuation required $1200 \mathrm{~mA}$. For a typical cantilever type device with a $0.87 \Omega$ coil, the actuation power (i.e., power consumed by the coil) was $320 \mathrm{~mW}$ at the $600 \mathrm{~mA}$ actuation current.

Measurements of the microrelay contact resistance under magnetic actuation were performed in air using a DC multimeter. "ON" resistances as low as $0.85 \Omega$, with typical values of 5 $10 \Omega$, were seen repeatedly. These results were observed on several different relay designs, including cantilever and bridge suspensions. The "OFF" resistance was infinite; no current flow was observed with voltages in excess of $10 \mathrm{~V}$ applied across the contacts.

A long-term reliability test of the relay was performed using a computer-controlled actuation and measurement setup. Figure 6 shows reliability test results for a typical cantilevertype device. In Figure 6, the "ON" resistance of the relay as a function of number of trials is shown. For the first 9,000 trials, the device is operating in the first mode (plate bridging two contacts); for the second 8,000 trials, the device is operating in the second mode (plate acting as one contact); the shift in contact resistance between mode operation shows the importance of the contact surfaces on the contact resistance of the microrelay. A vertical line extending to the top of the graph indicates an open circuit when current is applied to the driving coils, i.e., a failure of the relay to close. After an initial burn-in period, the device showed more than 14,000 operations with only one missed switching operation (defined as a contact resistance in the "ON" state of more than $300 \Omega$ ). Above 15,000 operations, several more failures were observed. The test was arbitrarily terminated at 17,000 operations, at which time the relay was still functional.

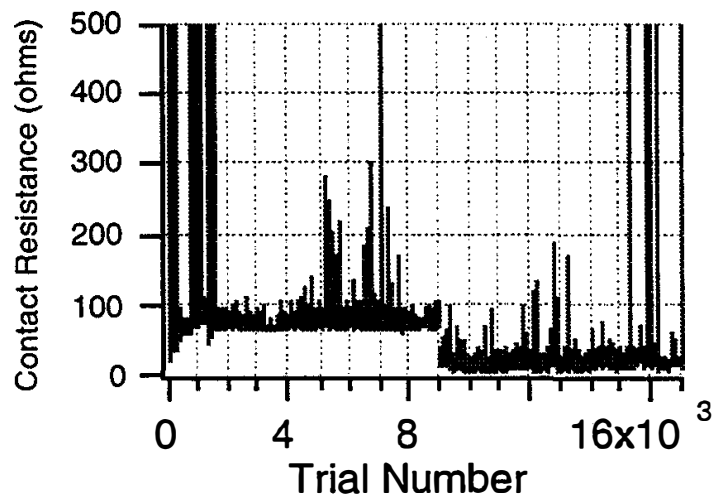

Figure 6: Lifetime results for a cantilever beam upper magnetic plate relay. The actuation current was $600 \mathrm{~mA}$.

Testing the relay with an active load which could be varied between 10 and $60 \mathrm{~mA}$ has also been performed. A cantilever type relay operating at $600 \mathrm{~mA}$ coil current was able to repeatedly switch a $10 \mathrm{~mA}$ DC load. The maximum load switched by any relay design was $60 \mathrm{~mA}$ at a coil current of 800 $\mathrm{mA}$. The actuation time was measured to be less than $10 \mathrm{msec}$. Release times were measured to be $25 \mathrm{msec}$. Localized contact damage was observed upon application of a $30 \mathrm{~V} \mathrm{DC}$ signal. The contacts when closed mechanically (non-switching) had a contact resistance of $0.017 \Omega$ and could carry 4.5 A of current for 2 minutes before failure occurred, and 3 A repeatedly without failure. Although these large carry currents have not yet been switched, it should be noted that these carry currents are well above the maximum $1 \mathrm{~A}$ of conventional reed relays, and comparable to small size armature relays.

\section{CONCLUSIONS}

A fully integrated magnetically actuated micromachined relay has been fabricated and tested. Actuation of the microrelay occurs at $600 \mathrm{~mA}$ coil current, $320 \mathrm{~mW}$ coil power for a cantilever type upper plate. These coil power levels are comparable with armature relays, and within a factor of ten when compared to typical reed relays. The contact resistance of the microrelay is less than $30 \Omega$. Preliminary lifetime tests show that the microrelay can operate over 14,000 cycles with only one failed switching action. The microrelay has shown the ability to repeatedly switch up to $60 \mathrm{~mA}$ electromagnetically, and in excess of $3 \mathrm{~A}$ with mechanical actuation.

In these prototype microrelays no attempt has been made to address contact issues. In addition, the coil has not been optimized for minimum resistance. The resultant improvement in efficiency when the coil and contacts are optimized may lead to fully integrated magnetically actuated micromachined relays with characteristics that compare favorably to conventional reed and armature devices.

\section{REFERENCES}

1. K. E. Petersen, "Micromechanical Membrane Switches on Silicon," IBM J. Res. Develon. vol. 23, no. 4, 376-385 (1979)

2. M. A. Gretillat, P. Thieubaud, N. F. de Rooij, and C. Linder, "Electrostatic Polysilicon Microrelays Integrated with MOSFETs," Proc. IEEE Microelectromechanical Sys. Conference, Oiso, Japan, pp. 97-101 (1994).

3. M. A. Gretillat, P. Thieubaud, C. Linder, and N. F. de Rooij, "Integrated circuit compatible electrostatic polysilicon microrelays," J. Micromech. Microeng., 5, 156-160 (1995).

4. S. Roy and M. Mehregany, "Fabrication of electrostatic nickel microrelay by surface micromachining," Proc. IEEE Microelectromechanical Sys. Conference Amsterdam, Netherlands, pp. 353-357 (1995).

5. J. Drake, H. Jerman, B. Lutze, and M. Stuber, "An Electrostatically Actuated Microrelay," Proc. Transducers '95 Stockholm, Sweden, vol. 2, pp. 380-383 (1995).

6. M. Sakata, "An Electrostatic Microactuator for ElectroMechanical Relay," Proc. IEEE MEMS Workshon. Salt Lake City, 149-151 (1989).

7. J. J. Yao and M. F. Chang, "A Surface Micromachined Miniature Switch for Telecommunications Applications with Signal Frequencies up to 4GHz," Proc. Transducers '95. Stockholm, Sweden, vol. 2, pp. 384-387 (1995).

8. J. Simon, S. Saffer, and C.J. Kim, "A Micromechanical Relay with a Thermally-Driven Mercury Micro-Drop," Proc. IEEE Microelectromechanical Sys. Conference, San Diego, pp. 515520 (1996).

9. H. Hosaka, H. Kuwano, and K. Yanagisawa, "Electromagnetic Microrelays: Concepts and Fundamental Characteristics," Proc. IEEE Microelectromechanical Sys. Conference. Ft. Lauderdale, pp. 12-17 (1993).

10. E. Hashimoto, H. Tanaka, Y. Suzuki, Y. Uenishi, and A. Watabe, "Thermally Controlled Magnetization Actuator (TCMA) using Thermosensitive Magnetic Materials," Proc. IEEE Microelectromechanical Sys. Conference, Oiso, Japan, pp. 108-113 (1994).

11. H. Hosaka, H. Kuwano, and K. Yanagisawa, "Electromagnetic microrelays: consepts and fundamental characteristics," Sensors and Actuators A, vol. A40, 41-47, (1994).

12. A. B. Frazier, C. H. Ahn, and M. G. Allen, "Development of Micromachined Devices Using Polyimide-Based Processes, Sensors and Actuators A (Physical), vol.A45, no.1, 47-55, (1994). 